\title{
Komplizierte Koexistenzen: IPF plus Lungenkarzinom - OSA plus Rheuma
}

\author{
Gesellt sich zu einer Erkrankung eine weitere, wird das Management nicht einfacher. Bei \\ idiopathischer Lungenfibrose drohen vermehrt Lungenkarzinome. Eine obstruktive Schlafapnoe \\ scheint mit rheumatischen Erkrankungen assoziiert. Eine solche Koexistenz hat Konsequenzen.
}

„Läuse und Flöhe zu haben, ist keine gute Idee“, konstatierte Prof. Antje Prasse von der Medizinischen Hochschule Hannover und machte das deutlich an der Koexistenz zwischen idiopathischen Lungenfibrose (IPF) und Lungenkrebs. Das Risiko für Patienten mit IPF zusätzlich an Lungenkrebs zu erkranken, ist hoch. Innerhalb von zehn Jahren nach Diagnosestellung entwickeln $60 \%$ der IPF-Patienten ein Karzinom. Die Karzinome entstehen mehr peripher und in den Fibrosezonen der Lunge. Sehr häufig handelt es sich um periphere Plattenepithelkarzinome. Liegt neben der IPF noch ein Emphysem vor, steigt das Risiko weiter. Doch nicht nur das Risiko für Lungenkrebs ist bei gleichzeitiger IPF erhöht. Auch die Prognose ist deutlich schlechter. Umgekehrt beeinflusst ein Lungenkarzinom den Verlauf der IPF nicht. „Die Prognose wird durch das Lungenkarzinom getrieben", so Prasse.

Pathophysiologischer Hintergrund für das gehäufte gemeinsame Auftreten ist ein Overlap zwischen Mechanismen, die die Entwicklung von IPF und Lungenkarzinom triggern. Die Risikofaktoren unter einer IPF ein Karzinom zu entwickeln, entsprechen denen bei Patienten ohne Karzinom: ein Alter über 60 Jahren (OR: 2,41), männliches Geschlecht (=R: 11,04) und Rauchen (OR:2,71). „Rauchen ist auch hier ein wesentlicher Risikofaktor, vor allem, wenn die Patienten noch aktiv rauchen. Umgekehrt haben Patienten mit IPF, die nie geraucht haben, ein sehr geringeres Risiko für ein Lungenkarzinom“, erläuterte Prasse.

\section{Checkpoint-Inhibitoren: „eine gute Idee“}

Ungünstiger ist bei gleichzeitiger IPF auch das therapeutische Outcome der Lungenkrebs-Therapie. Das gilt zum einen nach chirurgischer Resektion mit erhöhter Mortalität (46 vs. 20\%) und vermehrten postoperativen pneumologischen Komplikationen (27 vs. $18 \%$ ) ( Lee T et al. Resp Med 2014;108:1549-55). Des Weiteren besteht nach Radiotherapie u. a. ein erhöhtes Pneumonierisiko; Chemotherapie assoziiert ist vor allem die Verschlechterung der Lungenfunktion. Zu Checkpoint-Inhibitoren in dieser Konstellation gibt es noch keine Daten. Grundsätzlich ist die Checkpoint-Blockade aus Sicht von Prasse aber „eine gute Idee“, da auch bei der IPF das Immunsystem downreguliert ist.

\section{Bei Rheuma gezielt nach obstruktivem Schlafapnoe- Syndrom (OSA) fahnden}

Noch weniger gut untersucht ist die Koexistenz von OSA und Rheuma. Doch ebenfalls von Relevanz wie Dr. Andrea BosseHenk, Leiterin des Schlaflabors an der Med. Klinik I der Universität Leipzig, deutlich machte. Gesellt sich zu einer rheumatischen Erkrankung ein OSA, steigere dies Schmerz, Fatigue und das kardiovaskuläre Risiko. Per Fragebogen fand sich immerhin bei $35,2 \%$ von 400 Patienten mit rheumatischer Erkrankung ein hohes OSA-Risiko. Und polysomnografische Studien weisen auf eine erhöhte Prävalenz von bis zu $46 \%$ hin. Bosse-Henk forderte deshalb, Rheumapatienten systematisch zu ihrem Schlaf zu befragen, „denn für OSA gibt es gute Therapieoptionen.“

\section{Welche Rolle spielen proinflammatorische Zytokine?}

Wie genau die Zusammenhänge zwischen Schlaf, Schmerz und Entzündung sich äußern, ist noch unklar. Ein Zusammenhang scheint auf der Ebene der Entzündungsmarker zu bestehen. So waren bei Probanden mit Schlafentzug und Patienten mit OSA das CRP und proinflammatorische Zytokine erhöht. Von 63 Männern mit Spondylarthritis hatten 45 (76\%) ein OSA, davon bei $57 \%$ unter einem TNF-alpha-Inhibitor, aber bei $91 \%$ ohne TNF-alpha-Inhibitor (Walsh JA et al. J Clin Sleep Med 2012; 8(6) 643-8). Eine n-CPAP-Therapie führt zu einem Rückgang von TNF-alpha. Vermutet wird, dass Gelenkdeformitäten ein OSA triggern, etwa durch Bewegungseinschränkungen oder Instabilität der HWS. Bei anterioren atlantoaxialen Subluxationen mit zervikaler Myopathie kann es im Falle einer Kompression des Hirnstammes zu einer Beeinträchtigung des Atemzentrums mit Ausbildung einer zentralen Schlaf-Apnoe kommen. Beate Fessler

Quelle: 57. Kongress der Deutschen Gesellschaft für Pneumologie, Leipzig, 4. März 2016 